THEOREM 11. If $(a, b)=1$, and $m$ is the product of the distinct prime factors of $n$, then

$$
\left(n^{3} / m\right) \mid \phi\left(a^{2 n}-b^{2 n}\right) .
$$

CoRnell University

\title{
APPLICATIONS OF TRANSITIVITIES OF BETWEENNESS IN LATTICE THEORY ${ }^{1}$
}

\author{
M. F. SMILEY AND W. R. TRANSUE
}

Introduction. This paper solves three characterization problems for lattices $^{2}[1]$. Problem I is to characterize those metric spaces [2] into which lattice operations which are consistent with the given metric $[1$, p. 41] may be introduced. Problem II is to characterize those members of a rather general class of abstract systems which are modular lattices, while Problem III consists in the characterization of lattices in an even larger class of abstract systems:

Problem I has already been solved by V. Glivenko [3]. He showed that the property: "Among those elements metrically between $[4$, p. $76 ; 2$ ] two elements $a$ and $b$, the element $a \cup b$ is farthest from $O$," and its dual characterize those metric spaces which are also metric lattices with the same metric and least element $O$. Our approach to Problem I is through the existence of certain metric singularities [2, p. 47] in every metric lattice. Our solution also involves certain five point transitivities [5, Part I] of metric betweenness. The abstract system involved in Problem II (Problem III) is a wide generalization of the concept of a metric space-so general, in fact, that it also includes the concept of a modular lattice (lattice). We find it not difficult to extend the ideas essential to our solution of Problem I to give analogous solutions of Problems ${ }^{3}$ II and III. Briefly, our results consist in characterizing the three important systems: metric

Presented to the Society, April 4, 1942; received by the editors June 2, 1942.

1 This paper is an expansion of our note Metric lattices as singular metric spaces which was presented to the Society on December 29,1941, and which was to appear in Bull. Amer. Math. Soc.

2 Numbers in square brackets refer to the list of references at the end of the paper.

${ }^{3}$ It would be interesting to characterize metric spaces among our general systems. This problem appears difficult to us, and we make no attempt to solve it. 
lattices, modular lattices, and lattices within three increasingly general abstract systems.

1. A characterization of metric lattices. In this section we shall give our solution of Problem I. The metric singularities which we shall encounter are pseudo-linear quadruples [2, p. 48]. Such a quadruple is one which cannot be imbedded in the Euclidean line, but is such that each of the four triples chosen from it can be so imbedded. In metric lattices such configurations abound. Every pair of elements which are not comparable together with their meet and join form such a quadruple. But the existence of "sufficiently many" pseudolinear quadruples in a metric space $(M, \delta)$ is not enough to ensure that lattice operations can be introduced in such a way that $(M, \delta)$ becomes a metric lattice $(M, \delta,<)$. However, the additional assumption of a weak form of either of two five point transitivities for the metric betweenness of $(M, \delta)$ suffices.

Before we proceed to prove these statements, let us agree on the following matters of notation. The symbols $(M, \delta)$ represent a metric space $M$ with metric $\delta$. For elements $a, b, c \in M$ we use the notation $a b c$ to indicate that $b$ is metrically between $a$ and $c[4$, p. $75 ; 5$, Part II], that is, that $\delta(a, c)=\delta(a, b)+\delta(b, c)$. This relation has the fundamental properties :4

$$
\begin{gathered}
a b c \leftrightarrow c b a . \\
a b c \cdot a c b \leftrightarrow b=c .
\end{gathered}
$$

We shall frequently refer to the transitivities:

$$
\begin{aligned}
& a b c \cdot a d b \rightarrow d b c . \\
& a b c \cdot a d b \rightarrow a d c .
\end{aligned}
$$

which are valid for the metric betweenness of $M[4$, p. 76; 5, Part II $]$. If $(M, \delta)$ is also a metric lattice $(M, \delta,<)$, then the relation $a b c$ is equivalent [3] to the equations

$$
(a \cap b) \cup(b \cap c)=b=(a \cup b) \cap(b \cup c) .
$$

These equations define, in fact, a relation of betweenness in arbitrary lattices [5, Part II]. We shall also use the notation $a b c$ to indicate that the equations (1.1) are valid, even when no metric enters the

\footnotetext{
${ }^{4}$ The second of these statements is intended to be read as, "For each three elements $a, b, c \in M$, the relations $a b c$ and $a c b$ hold if and only if $b=c$." This convenient notation was used with profit in [5]. We shall attempt to preserve as many of the other notations introduced in [5] as possible.
} 
discussion. In this paper we shall need the fact that this relation of lattice betweenness satisfies $(\alpha),(\beta),\left(t_{1}\right)$, and

$$
a b c \rightarrow a \cap c \leqq b \leqq a \cup c .
$$

Further properties of this relation are given in [5, Part II ].

With reference to each point $p \in M$ of a metric space $(M, \delta)$ we may define a partial ordering of $M$ as follows [3].

If $a, b \in M$, then $a<_{p} b$ in case pab and $a \neq b$. The transitivity of the relation $<_{p}$ follows from the transitivity $t_{2}$ of metric betweenness. The antisymmetry of $\leqq_{p}$ is apparent. We shall be interested in the following conditions [5, Part I].

$$
\begin{aligned}
& p b c \cdot p d c \cdot b x d \rightarrow p x c . \\
& p b c \cdot p b d \cdot c x d \rightarrow p b x .
\end{aligned}
$$

REMARK 1. Condition $\left(T_{5}^{p}\right)$ states that an upper bound for the set $[b, d]$ is greater than every element between $b$ and $d$, while condition $\left(T_{7}^{p}\right)$ states that a lower bound for the set $[c, d]$ is smaller than every element between $c$ and $d$.

We shall also need the following condition.

$\left(A^{p}\right)$ If $a, b \in M$ and if $a$ and $b$ are not comparable by the relation $\leqq_{p}$, then there are elements $a \cup b, a \cap b \in M$ for which we have $(a \cup b) a(a \cap b)$, $(a \cup b) b(a \cap b), a(a \cup b) b, a(a \cap b) b$, and $p(a \cap b)(a \cup b)$.

We may now state the following theorem.

TheOREM 1. Let $(M, \delta)$ be a metric space. If $O \in M$, then $\left(M, \delta,<_{0}\right)$ is a metric lattice with least element $O$ if and only if $(M, \delta)$ satisfies $\left(A^{0}\right)$ and $\left(T_{5}^{0}\right)$ or $\left(A^{0}\right)$ and $\left(T_{7}^{0}\right)$.

Proof. The necessity of the condition $\left(A^{0}\right)$ is obvious. But both $\left(T_{5}^{p}\right)$ and $\left(T_{7}^{p}\right)$ also hold in every lattice if $p$ is a distributive element. We prove this in the following lemma which is a generalization of Theorem 9.4 of [5].

LEMma 1. If $L$ is a lattice and $p$ is a distributive element of $L$, then the conditions $\left(T_{5}^{p}\right)$ and $\left(T_{7}^{p}\right)$ are true.

Proof. We prove $\left(T_{5}^{p}\right)$ first. Let $p \in L$ be a distributive element of a lattice $L$, and consider elements $b, c, d, x \in L$ satisfying $p b c, p d c$, and $b x d$. From (1.2) we obtain $b \leqq p \cup c, d \leqq p \cup c$, and $x \leqq b \cup d$. Hence $x \leqq p \cup c$. Dually, $x \geqq p \cap c$. Since $p$ is distributive, it follows that $(p \cap x) \cup(x \cap c)=x \cap(p \cup c)=x$, and dually. Thus $\left(T_{5}^{p}\right)$ holds. To prove $\left(T_{7}^{p}\right)$, let $p \in L$ be a distributive element and consider elements $b, c, d, x \in L$ satisfying $p b c, p b d$, and $c x d$. As before, we have $b \leqq p \cup c$, 
$b \leqq p \cup d$, and $x \geqq c \cap d$. Consequently, since $p$ is distributive, $p \cup x$ $\geqq p \cup(c \cap d)=(p \cup c) \cap(p \cup d) \geqq b$. Dually, $p \cap x \leqq b$. Another application of the distributivity of $p$ yields $(p \cap b) \cup(b \cap x)=b \cap(p \cup x)=b$. By duality we obtain the relation $p b x$. Thus $\left(T_{7}^{p}\right)$ also holds and the proof of the lemma is complete.

The necessity of the conditions $\left(T_{5}^{0}\right)$ and $\left(T_{7}^{0}\right)$ now follows from Lemma 1 and the fact that the least element of a lattice is a distributive element.

We pass to the proof of the sufficiency of our conditions. Using only $\left(A^{o}\right)$ we see that $a \cup b$ and $a \cap b$ are upper and lower bounds, respectively, for the set $[a, b]$. This follows on applying the transitivities $t_{1}$ and $t_{2}$ to the relations $(a \cup b) a(a \cap b),(a \cup b)(a \cap b) O$, and to the relations $(a \cup b) b(a \cap b),(a \cup b)(a \cap b) O$. The force of the conditions $\left(T_{5}^{0}\right)$ and $\left(T_{7}^{O}\right)$ is that each ensures that $a \cup b$ and $a \cap b$ are unique least upper and greatest lower bounds, respectively. We shall give the details of the proof only in the case in which $\left(A^{\circ}\right)$ and $\left(T_{5}^{0}\right)$ are assumed. The other set of conditions may be handled by almost identical arguments. Hence suppose that the conditions $\left(A^{o}\right)$ and $\left(T_{5}^{o}\right)$ are valid. We prove first that $a \cup b$ is the least upper bound of the set $[a, b]$ if $a$ and $b$ are not comparable. For, if $z \geqq a, b$ by definition we have $O a z$ and $O b z$. Condition $\left(A^{o}\right)$ yields $a(a \cup b) b$, and condition $\left(T_{5}^{o}\right)$ then gives $O(a \cup b) z$, that is, $z \geqq a \cup b$. The treatment of $a \cap b$ is less trivial. Consider an element $w \in M$ for which $w \leqq a, b$; that is, for which $O w a$ and $O w b$. We show first that $w>a \cap b$ is impossible as follows. If it were true we should have $O(a \cap b) w$, and this with $O w a$ and $O w b$ would yield, via $t_{1}$, the relations $(a \cap b) w a$ and $(a \cap b) w b$. Combining $a w(a \cap b)$ with $a(a \cap b) b$ we would obtain, again by $t_{1}$, the relation $w(a \cap b) b$. From this and the relation $(a \cap b) w b$ we would get $(a \cap b) w(a \cap b)$. But this last relation implies that $w=a \cap b$, which is contrary to our assumption. Thus the relation $w>a \cap b$ cannot hold. To show that $w \leqq a \cap b$ we again use indirect proof. If this relation fails to hold, then, since $w \ngtr a \cap b$, the elements $w$ and $a \cap b$ would not be comparable. The condition $\left(A^{o}\right)$ then implies the existence of elements $w \cup(a \cap b)$ and $w \cap(a \cap b)$ satisfying with $w$ and $a \cap b$ the relations listed in $\left(A^{o}\right)$. The application of the condition $\left(T_{5}^{o}\right)$ to the relations $O(a \cap b) a$, Owa, and $(a \cap b)((a \cap b) \cup w) w$ gives the relation $O((a \cap b) \cup w) a$. Likewise we obtain $O((a \cap b) \cup w) b$. Applying the transitivity $t_{1}$ to $((a \cap b) \cup w)(a \cap b) O$ and $a((a \cap b) \cup w) O$ yields $a((a \cap b) \cup w)(a \cap b)$. Similarly, $b((a \cap b) \cup w)(a \cap b)$. Again, the relations $b((a \cap b) \cup w)(a \cap b)$ and $b(a \cap b) a$ would give, by $t_{1}$, the relation $a(a \cap b)((a \cap b) \cup w)$. A final application of the transitivity $t_{1}$ to the relations $a(a \cap b)((a \cap b) \cup w)$ and $a((a \cap b) \cup w)(a \cap b)$ would pro- 
duce the relation $(a \cap b)\left((a \cap b) \cup_{w}\right)(a \cap b)$. But this implies that $a \cap b=(a \cap b) \cup w$, contrary to our assumption that $w \leqq a \cap b$ fails to hold. We conclude that $w \leqq a \cap b$ and that $a \cap b$ is the unique greatest lower bound for the set $[a, b]$. We have now shown that the relation $<_{o}$ has the lattice property. It is an easy consequence of the condition $\left(A^{O}\right)$ that $\delta(O, a)$ is a sharply positive modular functional and that

$$
\delta(a, b)=\delta(a \cup b, O)-\delta(a \cap b, O),
$$

This completes the proof that $\left(M, \delta,<_{0}\right)$ is a metric lattice.

REMARK 2. To show that one of the conditions $\left(T_{5}^{O}\right)$ or $\left(T_{7}^{O}\right)$ is actually needed in Theorem 1 , we exhibit a metric space $(M, \delta)$ which satisfies the condition $\left(A^{\circ}\right)$ but for which the partial orderings $<_{q}$ fail to have the lattice property for every $q \in M$.

Let $M$ consist of the six points $O, a, b, c, d, I$, with the distances of distinct points (we omit $\delta$ ) as follows: $I a=I b=O c=O d=a b=c d=2$, $I O=3$, all other distances between distinct points equal to one. The condition $\left(A^{o}\right)$ is valid for $(M, \delta)$ because only the pairs $a, b$ and $c, d$ are not comparable by the relation $<_{o}$ and for these pairs the choices $c \cup d=I, c \cap d=a$ or $b, a \bigcap b=O$, and $a \cup b=c$ or $d$ are effective in the condition $\left(A^{o}\right)$. Thus $<_{o}$ is not a lattice ordering. By the symmetry of $M$ it will suffice to show that $<_{a}$ is not a lattice ordering to prove our assertion completely. In $\left(M, \delta,<_{a}\right)$ we have, besides the obvious fact that $a$ is the least element, only the relations $O<b, c<b, d<b$, $c<I$, and $d<I$. This is obviously not a lattice ordering of $M$.

2. A characterization of modular lattices. We turn now to a consideration of Problem II. The abstract systems which we shall consider consist of a class $K$ of elements $a, b, c, \cdots$ together with a triadic relation $R$ defined on $K$ and satisfying $(\alpha),(\beta),\left(t_{1}\right)$, and $\left(t_{2}\right)$. We shall find conditions which are necessary and sufficient that such a system be a modular lattice whose lattice betweenness is identical with the relation $R$.

For an arbitrary triadic relation $R$ defined on a class $K$ of elements $a, b, c, \cdots$ we make the following definition.

(2.1) If $a, b \in K$ then $a<_{p} b$ in case $(p, a, b) R$ and $a \neq b$.

Using this notation, our solution of Problem II takes the following form.

THEOREM 2.1. If a triadic relation $R$ defined on a class $K$ satisfies $(\alpha),(\beta),\left(t_{1}\right)$, and $\left(t_{2}\right)$, then $\left(K, \leqq_{0}\right)$ is a modular lattice with least element $O$ if and only if $(K, R)$ satisfies $\left(A^{O}\right)$ and $\left(T_{5}^{O}\right)$ or $\left(A^{0}\right)$ and $\left(T_{7}^{O}\right)$. When either of these alternatives holds, the relation $R$ is identical with the lattice betweenness of $K$. 
Proof. We note first that if $\left(A^{0}\right)$ and $\left(T_{5}^{O}\right)\left(\left(A^{0}\right)\right.$ and $\left.\left(T_{7}^{O}\right)\right)$ hold for $R$, the proof of Theorem 1 assures us that $\left(K, \leqq_{0}\right)$ is a lattice. Notice also that $a \leqq_{0} b \leqq_{0} c$ implies that $(a, b, c) R$ holds by the definition of $\leqq_{0}$ and $t_{1}$. The truth of the second sentence of the theorem is then evident from the following theorem [5, Theorem 10.1].

THEOREM 2.2. If $L$ is a lattice and $R$ is a triadic relation defined on $L$, then $R$ is the relation of lattice betweenness of $L$ if and only if the following conditions hold.

(i) The relation $R$ satisfies $(\alpha),(\beta)$, and $\left(t_{1}\right)$.

(ii) If $a \leqq b \leqq c$, then we have $(a, b, c) R$.

(iii) If the relation abc holds, then in the sublattice generated by $a, b, c$, the transitivity $t_{2}$ holds for $R$.

That the modular law holds in $\left(K, \leqq_{0}\right)$ is now implied by $t_{2}[\mathbf{5}$, Theorem 9.1]. This completes the proof that $\left(A^{0}\right)$ and $\left(T_{5}^{O}\right)\left(\left(A^{0}\right)\right.$ and $\left.\left(T_{7}^{O}\right)\right)$ are sufficient. Their necessity follows from Theorem 2.2 and Lemma 1. The proof of Theorem 2.1 is now complete.

3. A characterization of lattices. In this final section we shall present our solution of Problem III. The abstract systems which we shall consider consist of a class $K$ of elements $a, b, c, \cdots$ together with a triadic relation $R$ defined on $K$ and satisfying $(\alpha),(\beta)$, and $\left(t_{1}\right)$. We shall find sufficient conditions that such a system be a lattice under an ordering given by (2.1). In the presence of these conditions, we shall find necessary and sufficient conditions that the postulated relation $R$ coincide with the lattice betweenness of $K$. The first of these results is given in the following theorem.

THEOREM 3.1. If a triadic relation $R$ defined on a class $K$ satisfies $(\alpha),(\beta)$, and $\left(t_{1}\right),\left(A^{0}\right)$ and $\left(T_{5}^{0}\right)$, then the relation $\leqq_{0}$ is a partial ordering of $K$ with the lattice property.

Proof. The antisymmetry of $\leqq_{0}$ is a consequence of $(\beta)$, while the transitive law for $\leqq_{0}$ may be easily obtained if we notice that identifying $d$ and $O$ in $\left(T_{5}^{O}\right)$ yields the condition

$$
(O, b, c) R \cdot(O, x, b) R \rightarrow(O, x, c) R .
$$

We may also note that identification of $c$ and $d$ in $\left(T_{5}^{0}\right)$ yields the condition

$$
(O, b, c) R \cdot(b, x, c) R \rightarrow(O, x, c) R .
$$

This last condition may be used to replace $t_{2}$ in the proof given in Theorem 1 of the sufficiency of the conditions $\left(A^{0}\right)$ and $\left(T_{5}^{0}\right)$. A careful 
examination of our proof will reveal that $t_{2}$ was used only to show that the relations $(O, a, a \cup b) R$ and $(O, b, a \cup b) R$ follow from $(O, a \cap b, a \cup b) R$, $(a \cap b, a, a \cup b) R$ and $(a \cap b, b, a \cup b) R$. But it is easy to see that these conclusions follow from the same hypotheses under condition (3.1) as well as under $t_{2}$. We can then follow the proof of Theorem 1 from this point until the conclusion that $\left(K, \leqq_{0}\right)$ is a lattice has been reached. The proof of Theorem 3.1 is complete.

REMARK 3. That the sufficient conditions of Theorem 3.1 are not necessary conditions can be seen by the following example. Let the class $K$ consist of the five points $O, a, b, c, I$, and let the relation $R$ hold for the triples $O a I, O b I, O c I, O b c$, and $b c I$, as well as for those then required by $(\alpha)$ and $(\beta)$. It is easy to verify that $t_{1}$ holds in this example while $\left(A^{o}\right)$ fails. But $R$ gives rise through $\leqq_{0}$ to the simplest non-modular lattice.

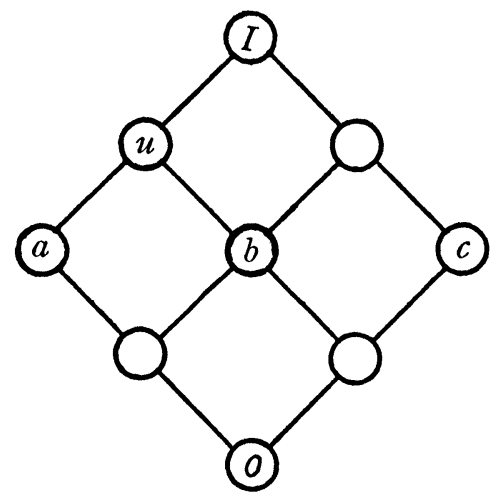

Fig. 1

Remark 4. Theorem 3.1 leaves us in doubt as to the question of the identity of the triadic relation $R$ and lattice betweenness in $K$. The following example shows that still further assumptions must be made if we are to obtain this conclusion. Let $K$ consist of the elements of the lattice shown in Figure 1. Define $R$ to be lattice betweenness except that the relation $(a, b, c) R$ is rejected. That $R$ satisfies the conditions $(\alpha)$ and $(\beta)$ is clear since they hold for lattice betweenness and their conclusions do not involve three distinct elements. To verify that $t_{1}$ holds for $R$ we must make sure that we cannot arrange its hypotheses so as to obtain $(a, b, c) R$ in the conclusion. To obtain $(a, b, c) R$ from $t_{1}$ would require hypotheses of the form $(d, b, c) R$, $(d, a, b) R$ or of the form $(d, b, a) R,(d, c, b) R$. But these sets cannot hold in our example, since if we have $(d, a, b) R$ and $(d, b, c) R$, then $d \neq a$, and $d \cap b \leqq a \leqq d \cup b$, by (1.2). It follows that $d \cup b=u$ or $I$, con- 
trary to $d \cap b \leqq a$. The other set of hypotheses may be treated similarly by interchanging $a$ and $c$. It is also easy to check that $R$ satisfies $\left(A^{O}\right)$. It remains only to remark that the conclusion of $\left(T_{5}^{0}\right)$ cannot be $(a, b, c) R$ (since $O$ must appear in the conclusion of $\left(T_{5}^{O}\right)$ ) to assure ourselves that $R$ satisfies all of the conditions of Theorem 4 . Thus $R$ generates through $\leqq_{0}$ the lattice of Figure 1 but $R$ is not the relation of lattice betweenness of this lattice.

To overcome this difficulty we propose the following strengthened condition.

(3.2) If $(p \cap x) \cup(x \cap c)=x \cap(p \cup c)$ and dually, then

$$
(p, b, c) R \cdot(p, d, c) R \cdot(b, x, d) R \rightarrow(p, x, c) R .
$$

The proof of Lemma 1 shows that (3.2) holds for lattice betweenness in arbitrary lattices.

We conclude with the following theorem.

THEOREM 3.2. If a triadic relation $R$ defined on a class $K$ satisfies $(\alpha),(\beta),\left(t_{1}\right),\left(T_{5}^{0}\right)$, and $\left(A^{0}\right)$, then $R$ is the lattice betweenness of the lattice $\left(K, \leqq_{0}\right)$ if and only if $R$ satisfies (3.2).

Proof. The remark preceding the statement of the theorem disposes of the necessity of (3.2). To establish the sufficiency we rely on the proof of Theorem 10.1 of [5] to assure us that the relation $(a, b, c) R$ implies $a b c$ (lattice betweenness). It remains, then, to prove that the relation $a b c$ implies the relation $(a, b, c) R$. It was proved in [5] that when $a b c$ holds the sublattice generated by $a, b, c$ is distributive. Hence the transitivity $\left(T_{5}\right)$ (see [5]) is available to us in this sublattice by virtue of (3.2). Now notice that since $a \cup c \geqq_{0} b \geqq_{0} a \cap c$ (by (1.2)), we have $a \cup b \cup_{c}=a \cup_{c}$ and dually. The condition $\left(A^{0}\right)$ then gives the relations $(a, a \cup b \cup c, c) R$ and $(a, a \cap b \cap c, c) R$. Clearly $a \cup b \cup c \geqq_{0} b \geqq_{0} a \cap b \cap c$; from which we obtain $(a \cup b \cup c, b, a \cap b \cap c) R$. The transitivity $\left(T_{5}^{o}\right)$ then yields $(a, b, c) R$ as desired and the proof is complete.

\section{REFERENCES}

1. G. Birkhoff, Lattice theory, Amer. Math. Soc. Colloquium Publications vol 25, 1940.

2. L. M. Blumenthal, Distance geometries, University of Missouri Studies vol. 13 (1938) no. 2.

3. V. Glivenko, Géométrie des systèmes de choses normées, Amer. J. Math. vol. 58 (1936) pp. 799-828.

4. K. Menger, Untersuchungen ïber die allgemeine Metrik, Math. Ann. vol. 100 (1928) pp. 75-163.

5. Everett Pitcher and M. F. Smiley, Transitivities of betweenness, Trans. Amer. Math. Soc. vol. 52 (1942) pp. 95-114.

LEHIGH UNIVERSITY 